\title{
Sorafenib in metastatic thyroid cancer
}

\author{
Jaume Capdevila, Lara Iglesias ${ }^{1}$, Irene Halperin ${ }^{2}$, Ángel Segura ${ }^{3}$, \\ Javier Martínez-Trufero, Maria Ángeles Vaz ${ }^{4}$, Jesús Corral ${ }^{6}$, \\ Gabriel Obiols ${ }^{7}$, Enrique Grande ${ }^{5}$, Juan Jose Grau ${ }^{8}$ and Josep Tabernero
}

\author{
Medical Oncology Department, Vall d'Hebron University Hospital, Universitat Autònoma de Barcelona, P. Vall d'Hebron 119-129, \\ 08035 Barcelona, Spain \\ ${ }^{1}$ Medical Oncology Department, Hospital Universitario 12 de Octubre, Madrid, Spain \\ ${ }^{2}$ Endocrinology Department, Hospital Clinic, Barcelona University, Barcelona, Spain \\ ${ }^{3}$ Medical Oncology Department, La Fe University Hospital, Valencia, Spain \\ ${ }^{4}$ Medical Oncology Department, Hospital Miguel Servet, Zaragoza, Spain \\ ${ }^{5}$ Hospital Universitario Ramón y Cajal, Madrid, Spain \\ ${ }^{6}$ Medical Oncology Department, Hospital Universitario Virgen del Rocío, Sevilla, Spain \\ ${ }^{7}$ Endocrinology Department, Vall d'Hebron University Hospital, Barcelona University, Barcelona, Spain \\ ${ }^{8}$ Medical Oncology Department, Hospital Clinic, Barcelona, Spain \\ (Correspondence should be addressed to J Capdevila; Email: jacapdevila@vhebron.net)
}

\begin{abstract}
Although thyroid cancer usually has an excellent prognosis, few therapeutic options are available in the refractory setting. Based on the recent results of phase II studies with tyrosine kinase inhibitors, we designed a retrospective analysis of patients with metastatic thyroid cancer treated with sorafenib in seven Spanish referral centers. Consecutive patients with progressive metastatic thyroid cancer (papillary, follicular, medullary, and anaplastic) not suitable for curative surgery, radioactive-iodine therapy, or radiotherapy were treated with sorafenib $400 \mathrm{mg}$ twice a day. The primary end point was objective response rate (RR). Secondary end points included toxicity, median progression-free survival (mPFS), median overall survival (mOS), and correlation between tumor marker levels (thyroglobulin, calcitonin, and carcinoembryonic antigen) and efficacy. Between June 2006 and January 2010, 34 patients were included in the study. Sixteen patients presented differentiated thyroid carcinomas (DTC) of which seven (21\%) were papillary, nine $(26 \%)$ follicular, $15(44 \%)$ medullary (MTC), and three (9\%) were anaplastic (ATC). Eleven (32\%) patients achieved partial response and $14(41 \%)$ had stable disease beyond 6 months. Regarding histological subtype, RRs were $47 \%$ (seven of 15 ) for MTC, $19 \%$ (three of 16 ) for DTC, and $33 \%$ (one of three) for ATC. With a median follow-up of 11.5 months, mPFS were 13.5, 10.5, and 4.4 months for DTC, MTC, and ATC respectively. Tumor markers were evaluated in 22 patients, and a statistically significant association was observed between $\mathrm{RR}$ and decrease in tumor marker levels $>50 \%(P=0.033)$. In this retrospective trial, sorafenib showed antitumor efficacy in all histological subtypes of thyroid cancer, warranting further development in this setting.
\end{abstract}

Endocrine-Related Cancer (2012) 19 209-216

\section{Introduction}

Several translational and clinical studies have been designed based on the knowledge of the main molecular steps that lead to the transformation of the normal follicular cell into an invasive thyroid carcinoma, whose results are leading to a more optimistic outcome for the treatment of these patients. Thyroid cancer has become one of the most fascinating models of carcinogenesis and is a particularly promising paradigm for targeted therapy.
A multistep model has been proposed as a mechanism for the neoplastic process in thyroid cancer, involving the main genetic mutations that provoke the tumor-initiating process in a follicular cell, and the phenomenon of oncogenic addiction to these particular mutations that makes them particularly interesting for directed therapy (Puxeddu et al. 2011). One of the most important activating genetic mutations involves the RET/PTC-RAS-RAF-MAPK axis. For hereditary medullary thyroid cancer (MTC), mutations 
in the RET gene are essential in more than $95 \%$ of cases and are also important for the development of sporadic MTC (50\% of cases) (Nikiforova \& Nikiforov 2008). Activating mutations in protein kinase B-type RAF (BRAF) kinase play a key role in the carcinogenesis of papillary thyroid cancer (PTC) in up to $45 \%$ of cases, as do RAS mutations (10\%), as well as rearrangements in the RET gene (RET/PTC) in 5-30\% of cases. These mutations have also been described in follicular thyroid carcinomas (FTC) affecting, in more than $40 \%$ of cases, three genes in the $R A S$ proto-oncogene family (HRAS, NRAS, and KRAS). Genomic rearrangements occur in up to $50 \%$ of FTCs involving a translocation event between chromosome regions $3 \mathrm{p} 25$ and $2 \mathrm{q} 13$, which results in a fusion protein (paired box gene 8 (PAX8) with the peroxisome proliferatoractivated receptor $\gamma(P P A R \gamma(P P A R G))$ gene). Genetic alterations in the RAS-BRAF pathway have also been identified in poorly differentiated and anaplastic PTCs with the accumulation of other important protein mutations such as p53 (TP53), $\beta$-catenin, or phosphatidylinositol-3-kinase (PI3K (PIK3CA)). Additionally, upregulation of vascular endothelial growth factor (VEGF) and platelet-derived growth factor receptor (PDGFR (PDGFRB)) expression has been well described in thyroid cancer and correlated with tumor stage, size, and lymphatic and distant tumor spread with a detrimental impact on survival (Yu et al. 2005).

Sorafenib (BAY 43-9006) is the first compound capable of inhibiting all RAF kinases that are administered in clinical practice. Moreover, sorafenib targets a panel of tyrosine kinase receptors such as VEGF receptors 1-3, PDGFRB, and RET, which give sorafenib not only proapoptotic properties but also antiangiogenic effects that are of special interest in thyroid carcinomas (Wilhelm et al. 2004).

We report the results of a retrospective populationbased study evaluating the activity of sorafenib in patients with metastatic thyroid cancer.

\section{Materials and methods}

\section{Patients}

Eligible patients were $\geq 18$ years old with histologically confirmed thyroid carcinoma (papillary, follicular, medullary, or anaplastic subtypes) for which no curative or standard palliative therapies were available. Patients had evidence of disease progression by Response Evaluation Criteria in Solid Tumors (RECIST) in the 12 months before entering the study, and all patients had measurable disease according to RECIST criteria. Previous therapies such as radioiodine therapy, radiotherapy, chemotherapy, or biologic treatment were permitted. Other inclusion criteria included Eastern Cooperative Oncology Group performance status (ECOG) $\leq 2$ with preserved renal, hepatic, and bone marrow function. All patients provided written informed consent and were included in the Spanish compassionate use program. Also, authorizations from the hospitals' pharmacy departments, management, and central government for the off-label use sorafenib in patients with metastatic thyroid carcinoma were obtained for each patient.

\section{Study design}

This was a retrospective longitudinal study of a group of patients included in the Spanish off-label program of sorafenib for the treatment of metastatic thyroid carcinoma. Patients included in the analysis had received sorafenib $400 \mathrm{mg}$ twice a day as long as it provided clinical benefit or until unacceptable toxicity occurred. Patients with papillary, follicular, medullary, and anaplastic subtypes were included. The study was performed in accordance with local legislation in seven Spanish referral centers. Clinical benefit was defined as partial response (PR) or stable disease (SD). Patients were withdrawn from the study after radiologically confirmed tumor disease progression according to RECIST. Patients were evaluated clinically every month and radiologically every 2-3 weeks. A cycle was defined as 4 weeks, and tumor evaluation was performed by computed tomography (CT) scan or magnetic resonance imaging (MRI) every 8-12 weeks. Tumor marker evaluation, carcinoembryonic antigen (CEA), thyroglobulin, and calcitonin tests were performed every $8-12$ weeks. Dose adjustments were made according to toxicity profile.

\section{Study end points and statistical analysis}

The primary end point was efficacy evaluated in terms of response rate (RR) according to RECIST v1.0 (Therasse et al. 2000). Secondary end points included toxicity profile, evaluated by means of the Common Terminology Criteria for Adverse Events v3.0. (CTCAE v3.0), median progression-free survival (mPFS), median overall survival (mOS), and the correlation between efficacy and tumor marker levels: thyroglobulin for differentiated thyroid cancer (DTC) that included PTC and FTC, and calcitonin and CEA for MTC.

Statistical analyses of survival were performed using a log-rank of Kaplan-Meier survival estimates and $\chi^{2}$ distribution for the correlation between tumor markers and efficacy. 


\section{Results}

Between June 2006 and January 2010, 34 consecutive patients included in the Spanish off-label program of sorafenib for the treatment of metastatic thyroid carcinoma were included in the study, whose baseline characteristics are summarized in Table 1. Of the 34 patients with confirmed thyroid cancer, seven $(21 \%)$ were papillary, nine (26\%) follicular, $15(44 \%)$ medullary, and three (9\%) were anaplastic. At baseline, 33 patients $(97 \%)$ had previously undergone surgery, $19(56 \%)$ had received radiotherapy, 13 (38\%) radioactive iodine therapy, 11 (32\%) chemotherapy, seven $(20 \%)$ had received somatostatin analogs, and one patient $(3 \%)$ had received previous treatment with a tyrosine kinase inhibitor (TKI), namely sunitinib. All patients presented disease progression according to RECIST criteria before inclusion in the trial.

\section{Efficacy}

A total of 32 patients were evaluable for radiological response, but all 34 patients were included in the intention-to-treat analysis. Eleven patients reached PR and 14 reached SD beyond 6 months. The disease control rate $(\mathrm{PR}+\mathrm{SD})$ was $73 \%$. The $\mathrm{PR}$ rate by histological subtype was $7 / 15(47 \%)$ for patients with MTC, 3/16 (19\%) for patients with DTC, and 1/3 (33\%) for patients with anaplastic carcinomas. Disease control rate for patients with MTC was $87 \%$, and for patients with DTC, it was $69 \%$ (Table 2).

With a median follow-up of 11.5 months, mPFS was 10.5 months (95\% CI: 6.13-14.87) for the whole group of patients including all subtypes. As expected, mPFS differed according to thyroid carcinoma subtype (Fig. 1), from 4.4 months (95\% CI: 2.30-6.57) in the

Table 1 Patient characteristics

\begin{tabular}{ll}
\hline Total no. of patients & 34 \\
Gender M/F (no.) & $16 / 18$ \\
Median age (range) & 57.3 years (range 28.8-84.6) \\
ECOG/PS & $n(\%)$ \\
0 & $5(15)$ \\
1 & $20(59)$ \\
2 & $9(26)$ \\
Tumor subtypes & $n(\%)$ \\
Papillary & $7(23)$ \\
Follicular & $9(26)$ \\
Medullary & $15(44)$ \\
Anaplastic & $3(7)$ \\
Previous therapies & $n(\%)$ \\
Surgery & $33(97)$ \\
Radiotherapy & $19(56)$ \\
Radioiodine therapy & $13(38)$ \\
Chemotherapy & $11(32)$ \\
Somatostatin analogs & $7(20)$ \\
\hline
\end{tabular}

Table 2 Tumor response by histology subtypes

\begin{tabular}{llll}
\hline & \multicolumn{1}{c}{ PR } & \multicolumn{1}{c}{ SD } & PD \\
\hline Papillary & $14 \%(1 / 7)$ & $43 \%(3 / 7)$ & $43 \%(3 / 7)$ \\
Follicular & $22 \%(2 / 9)$ & $55 \%(5 / 9)$ & $11 \%(1 / 9)$ \\
DTC & $19 \%(3 / 16)$ & $50 \%(8 / 16)$ & $25 \%(4 / 16)$ \\
Medullary & $47 \%(7 / 15)$ & $40 \%(6 / 15)$ & $7 \%(1 / 15)$ \\
Anaplastic & $33 \%(1 / 3)$ & $0 \%$ & $66 \%(2 / 3)$ \\
\hline
\end{tabular}

anaplastic population to 13.3 months (95\% CI: $6.28-$ 20.45 ) in patients with DTC and 10.5 months (95\% CI: 4.49-16.51) in those with MTC. In terms of OS, the median for the whole group of patients was 23.6 months; when considering the different subtypes, the values were 5 months for the anaplastic tumor population and 23.6 months for the DTC population, although this value was not reached in patients with MTC (Fig. 2).

\section{Marker correlation}

Correlation between tumor marker decrease $>50 \%$ and tumor response to sorafenib was analyzed. Thyroglobulin was used for DTC and calcitonin and/or CEA for MTC. Twenty-eight (82\%) patients were evaluated for tumor marker response. In 16 patients, there was a decrease in any tumor marker $>50 \%$ from baseline value, eight of whom achieved PR. Of the 12 patients who did not show a decrease in tumor markers, only one patient presented a PR. A statistically significant association was observed between $>50 \%$ decrease of any tumor marker and the probability of achieving a tumor response according to RECIST criteria $\left(P<0.05, \chi^{2}\right.$ analysis). Analysis by tumor subtype and tumor marker type did not show statistical significance owing to the low number of patients in each group.

\section{Toxicity}

The most common side effects (frequency $\geq 5 \%$ ) were hand-foot syndrome, diarrhea, rash, asthenia, anorexia, stomatitis, hypertension, and abdominal pain. The majority of side effects were controlled with treatment delays or dose reductions. Table 3 summarizes all the toxicity profile observed during the study period. The toxicity profile was similar to that observed in patients with other primary tumors. Twelve (35\%) patients required a dose reduction due to toxicity. No patient required increased levothyroxine dose while on treatment with sorafenib. There was one death due to intracranial hemorrhage during the study, and a relationship with sorafenib cannot be ruled out. 


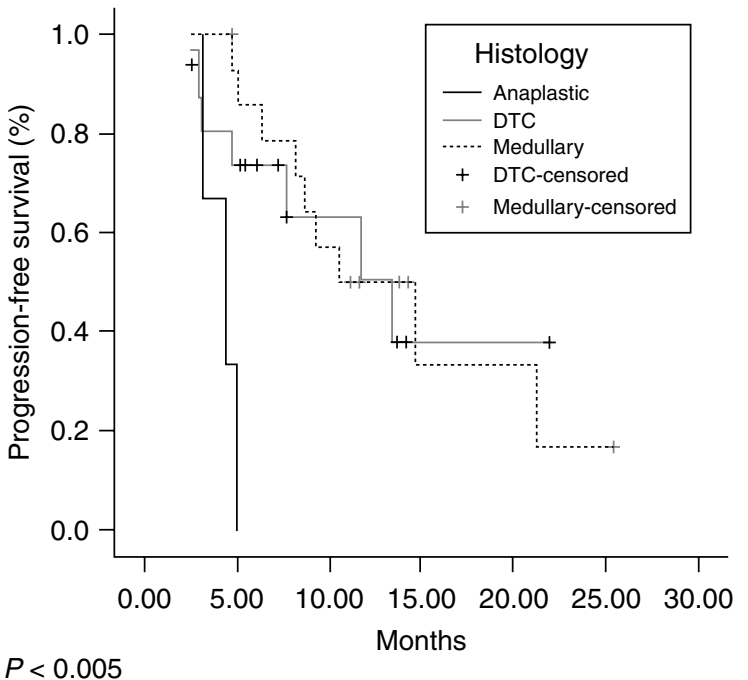

Figure 1 Median progression-free survival curves. Statistically significant differences were observed between DTC and anaplastic and also between medullary and anaplastic. No differences were observed for DTC and MTC.

\section{Discussion}

Until recently, medical management of advanced thyroid cancer refractory to standard treatments has been particularly challenging. Classical DNA-damaging drugs have demonstrated limited activity in this setting with no impact on survival. The knowledge of key mutational events in some genes including $B R A F$ and RET that affect early stages of thyroid carcinogenesis has opened up a new scenario for targeted therapies. Sorafenib is the only available drug that targets BRAF, RET, and VEGF receptors, which are involved in the pathogenesis of thyroid cancers, offering a strong molecular rationale for the treatment of all thyroid cancer subtypes.

In this retrospective study, we have reported the activity and safety of sorafenib in a group of patients with any subtype of thyroid cancer involved in an offlabel program of sorafenib for the treatment of metastatic thyroid carcinoma. We acknowledge that our study has important weaknesses, including its retrospective nature, the small sample size, and the lack of a control arm. Indeed, with this design, it is impossible to draw any definitive conclusions on the activity of sorafenib in patients with metastatic thyroid cancer, which can only be addressed by larger prospective randomized, placebo-controlled, clinical trials. Despite, we believe that one of the advantages of our trial is that the study population consisted of consecutive patients who had not been previously selected under the strict inclusion criteria of prospective trials, so the data obtained reflect the clinical situation in some ways. Also, by including the first 34 consecutive patients, the risk of retrospectively selecting the cases with better outcomes and the possibility of underestimating the occurrence of adverse events and overestimating the drug's efficacy are reduced.

The data obtained in our study are consistent with the data obtained from previous studies with sorafenib and also with other TKIs in patients with DTC in which the PR rate has ranged from 6 to $49 \%$ (19\% in this study), SD rates from 38 to $67 \%$ (50\% in our study), and mPFS from 15 to 20 months (13.3 months in our study). However, certain discrepancies such as initially documented disease progression status are seen that are likely related to different inclusion criteria between studies (Table 4). More recently, results of a phase II trial of pazopanib on patients with metastatic, rapidly progressive, radioiodine-refractory DTC showed a PR in 18 out of 39 patients, yielding a RR of $49 \%$ (95\% CI: 35-68) (Bible et al. 2010). Also, high RRs have been obtained in patients with DTC with newer compounds, including lenvatinib (E7080). In a phase II trial of lenvatinib by Sherman et al. (2011), confirmed PRs were observed in 29 of the 58 patients, yielding a RR of $50 \%$ (95\% CI: 37-63), and median PFS was 12.6 months (95\% CI: 10.4-14.1), similar to the PFS obtained in our study (13.3 months). A multicenter, randomized, double-blind, placebo-controlled, phase III trial is currently recruiting participants (NCT01321554). This trial is allowing the inclusion

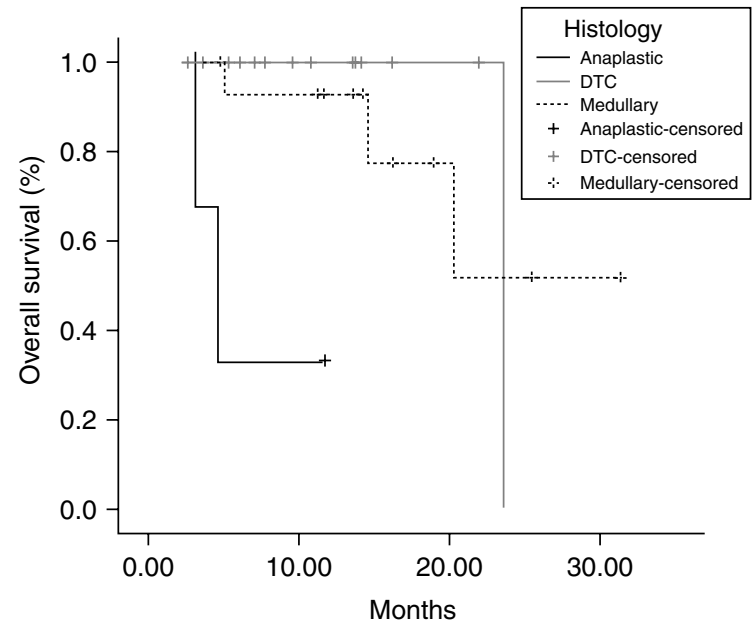

$P<0.005$

Figure 2 Median overall survival curves. Statistically significant differences were observed between DTC and anaplastic and also between MTC and anaplastic. No differences were observed between DTC and MTC. 
Table 3 Main side effects $(n=34)$

\begin{tabular}{llccc}
\hline & $\begin{array}{c}\text { Grade } \\
\mathbf{1}(\%)\end{array}$ & $\begin{array}{c}\text { Grade } \\
\mathbf{2}(\%)\end{array}$ & $\begin{array}{c}\text { Grade } \\
\mathbf{3}(\%)\end{array}$ & $\begin{array}{c}\text { Grade } \\
\mathbf{4}(\%)\end{array}$ \\
\hline $\begin{array}{l}\text { Hand-foot } \\
\quad \text { syndrome }\end{array}$ & $7(20)$ & $6(18)$ & $7(20)$ & $1(3)$ \\
Stomatitis & $6(18)$ & $9(26)$ & $1(3)$ & \\
Diarrheas & $6(18)$ & $10(29)$ & $5(15)$ & \\
Alopecia & $3(9)$ & $2(6)$ & $4(12)$ & \\
Hypertension & $4(12)$ & $2(12)$ & & \\
Abdominal pain & $5(15)$ & $5(15)$ & $1(3)$ & \\
Rash & $8(23)$ & $2(6)$ & $2(6)$ & \\
Dry mouth & $1(3)$ & $1(3)$ & & \\
Fatigue & $8(23)$ & $6(18)$ & $5(15)$ & \\
Anorexia & $1(3)$ & $3(9)$ & $3(9)$ & \\
Xerosis & $9(26)$ & $1(3)$ & $1(3)$ & \\
Dysgeusia & $1(3)$ & & & \\
Arthromyalgia & $1(3)$ & & & \\
Elevated & & & $1(3)$ & \\
$\quad$ transaminases & & & & \\
\hline
\end{tabular}

of patients that have received 0 or 1 before TKI, such as sorafenib, sunitinib, or pazopanib.

In a randomized, double-blind trial by Leboulleux et al., 145 patients with locally advanced or metastatic DTC were randomized to vandetanib $(n=72)$ or placebo $(n=73)$. Compared with placebo, vandetanib significantly improved PFS (11.0 vs 5.8 months; hazard ratio: $0.63 ; 95 \% \mathrm{CI}: 0.43-0.92 ; P=0.008$ ), which is similar to the PFS obtained in our study (13.3 months) (Leboulleux et al. 2010).
Currently, there is ongoing discussion about the best criteria to evaluate antitumor activity of targeted therapies, and it seems that RECIST criteria do not properly evaluate all the real benefits of new targeted agents in solid tumors. We chose RECIST v1.0 criteria instead of RECIST v1.1 criteria in an attempt to assess the maximum number of target lesions in the same organ. This contrasts with RECIST v1.1 criteria, which reduces the number of target lesions per organ and could subsequently jeopardize the best evaluation of a disease that usually metastasizes to one or two different organs with measurable disease (lymph nodes and lung) (Eisenhauer et al. 2009). The first international placebo-controlled phase III study with sorafenib in a population with DTC has recently completed its recruitment and will define the role of this TKI drug in the management of advanced radioiodine-refractory DTC (NCT00984282).

Data on sorafenib in MTC are limited compared with data on sorafenib in DTC. Other TKIs have demonstrated promising activity against MTC, such as cabozantinib (XL184), sunitinib, and motesanib (Table 4). Vandetanib, in particular, has recently received US Food and Drug Administration (FDA) approval for the treatment of advanced MTC after positive results from a placebo-controlled phase III study, which demonstrated a significant benefit in mPFS (hazard ratio: 0.46; 95\% CI: 0.31-0.69;

Table 4 Main studies in advanced thyroid cancer with targeted therapies

\begin{tabular}{|c|c|c|c|c|c|c|}
\hline References & Drug & $n$ & Tumor type & $\begin{array}{c}\text { Response } \\
\text { rate (\%) }\end{array}$ & $\begin{array}{c}\text { Stable } \\
\text { disease (\%) }\end{array}$ & $\begin{array}{l}\text { Median } \\
\text { progression- } \\
\text { free survival } \\
\text { (months) }\end{array}$ \\
\hline Kloos et al. (2009) & Sorafenib & 41 & DTC and ATC & 15 & 56 & 15 \\
\hline Gupta-Abramson et al. (2008) & Sorafenib & 30 & DTC & 23 & 34 & 20 \\
\hline Hoftijzer et al. (2009) & Sorafenib & 32 & DTC & 25 & 34 & 13.4 \\
\hline Ahmed et al. (2011) & Sorafenib & 26 & DTC and MTC & $18^{\mathrm{a}}$ & 82 & NR \\
\hline Sherman et al. (2008) & Motesanib & 93 & DTC & 14 & 67 & 9.2 \\
\hline Cohen et al. (2008) & Axitinib & 60 & All & 30 & 38 & 18.1 \\
\hline Ravaud et al. (2008) & Sunitinib & 17 & All & 30 & 38 & NR \\
\hline Carr et al. (2009) & Sunitinib & 33 & DTC and MTC & 32 & 48 & NR \\
\hline Bible et al. (2010) & Pazopanib & 37 & DTC & 49 & NR & 11.7 \\
\hline Leboulleux et al. (2010) & Vandetanib & 145 & DTC & 8.3 & $56.9^{\mathrm{b}}$ & 11 \\
\hline Sherman et al. (2011) & Lenvatinib & 58 & DTC & 59 & 36 & 13.3 \\
\hline De Souza et al. (2010) & Sunitinib & 25 & MTC & 33 & 54 & 12 \\
\hline Kurzrock et al. (2010) & $\mathrm{XL}-184$ & 37 & MTC & 29 & 41 & NR \\
\hline Wells et al. (2010) & $\begin{array}{c}\text { Vandetanib/ } \\
\text { placebo }^{c}\end{array}$ & 331 & MTC & 45 & NR & $\begin{array}{c}19.3 \text { vs not } \\
\text { reached }\end{array}$ \\
\hline Lam et al. (2010) & Sorafenib & 21 & MTC & 6.3 & 87.5 & 17.9 \\
\hline Schlumberger et al. (2009) & Motesanib & 91 & MTC & 2 & 81 & 11.1 \\
\hline
\end{tabular}

ATC, anaplastic thyroid cancer; DTC, differentiated thyroid cancer; MTC, medullary thyroid cancer; NR, not reported. 3-Month median follow-up.

${ }^{\mathrm{b}}$ Disease control rate.

cPhase III study. 
$P<0.001)$, objective RR $(P<0.001)$, and disease control rate $(P=0.001)$ (Wells et al. 2011).

Cabozantinib has also shown promising activity in a phase I dose escalation study carried out by Kurzrock et al. (2011). In this trial, ten out of 37 patients (29\%) with MTC obtained a PR. Moreover, preliminary results of the ongoing EXAM (Efficacy of cabozantinib (XL184) in Advanced Medullary Thyroid Cancer) trial (NCT00704730) have been reported (http://www. exelixis.com/pipeline/x1184), which show that cabozantinib produces a significant 2.8-fold increase in PFS compared with placebo (11.2 vs 4.0 months; hazard ratio: 0.28 ; 95\% CI: 95\% CI 0.19, $0.40 ; P<0.0001$ ).

The few data available on sorafenib in MTC patients showed limited activity with PR rates $<10 \%$ but higher SD rates especially in subgroup analyses of patients with tumor progression before study entry (Lam et al. 2010). Our data support the efficacy of sorafenib in a population of patients with MTC, achieving similar results compared with other TKIs in this setting (Table 4). Although trials are not directly comparable, it seems that different TKIs are able to provide significant benefit in MTC patients regardless of their affinity for wild-type or mutant RET receptors.

In ATC patients, the small sample number $(n=3)$ makes it difficult to extrapolate the data obtained from PR or SD. The observed results support a hypothesis of possible activity of TKIs in ATC. However, as some TKIs may need time to achieve active plasma concentrations to reduce tumor angiogenesis, these small molecules may not act as rapidly as necessary, and hence their use in ATC should be considered carefully.

The toxicity profile observed in this unselected cohort of patients was similar to that observed in previous studies and was managed mainly with both symptomatic treatment and sorafenib dose delay or reduction ( $35 \%$ in this study). It is of particular use to report the safety profile observed in routine clinical thyroid cancer patients, as toxicity data reported in patients in prospective clinical studies are often not taken from a representative sample. Although few patients presented grade 3-4-related toxicity, most patients presented some grade 1-2 side effects. For some of them, previously asymptomatic, this may represent a worsening in their quality of life that could jeopardize compliance to the treatment. Quality of life assessment is strongly recommended for future regulatory clinical trials.

With regard to tumor markers and their role in the evaluation of clinical efficacy, we observed that a reduction in CEA/calcitonin and thyroglobulin higher than $50 \%$ was correlated with PR. When we analyzed the data by tumor subtype and tumor marker type, the results were not significant due to the small number of patients. Data in this area are conflicting in phase II clinical trials and our results only suggest possible predictive biomarkers for tumor response. These results need to be confirmed in large prospective phase III clinical trials and should also provide information on the time to achieve marker response (not available in our study) in order to recognize, within a few weeks, those patients who are less likely to obtain objective response, which would shorten unnecessary drug exposure.

In summary, we report the efficacy and toxicity profile of sorafenib used off-label for the treatment of advanced and refractory thyroid cancer in an unselected population representative of routine clinical practice. The observed efficacy data are comparable to other published studies with sorafenib in DTC patients. However, we report a cohort of patients with MTC with interesting clinical activity warranting further development in this setting.

\section{Declaration of interest}

The authors declare that there is no conflict of interest that could be perceived as prejudicing the impartiality of the research reported.

\section{Funding}

This research did not receive any specific grant from any funding agency in the public, commercial, or not-for-profit sector.

\section{Author contribution statement}

$\mathrm{J}$ Capdevila and $\mathrm{J}$ Tabernero involved in the conception and design of the study. J Capdevila, L Iglesias, I Halperin, Á Segura, J Martínez-Trufero, M Á Vaz, J Corral, G Obiols, E Grande, and J J Grau provided the study materials and recruited the patients. J Capdevila and E Grande collected and assembled the data. All the authors analyzed and interpreted the data. All the authors involved in writing the manuscript and approved the final manuscript.

\section{Acknowledgements}

The authors acknowledge Gessamí Ollé and Debora Moreno for their statistical analysis support and Eric Johnson and Beatriz Gil-Alberdi for writing advice. The authors would like to express their gratitude to all patients and their families. 


\section{References}

Ahmed M, Barbachano Y, Riddell A, Hickey J, Newbold KL, Viros A, Harrington KJ, Marais R \& Nutting CM 2011 Analysis of the efficacy and toxicity of sorafenib in thyroid cancer: a phase II study in a UK based population. European Journal of Endocrinology 165 315-322. (doi:10.1530/EJE-11-0129)

Bible KC, Suman VJ, Molina JR, Smallridge RC, Maples WJ, Menefee ME, Rubin J, Sideras K, Morris JC III, McIver B et al. 2010 Efficacy of pazopanib in progressive, radioiodine-refractory, metastatic differentiated thyroid cancers: results of a phase 2 consortium study. Lancet Oncology 11 962-972. (doi:10.1016/S14702045(10)70203-5)

Carr L, Goulart B, Martins R, Keith E, Kell E, Wallace S, Capell P \& Mankoff D 2009 Phase II trial of continuous dosing of sunitinib in advanced, FDG-PET avid, medullary thyroid carcinoma (MTC) and welldifferentiated thyroid cancer (WDTC). ASCO Meeting Abstracts 276056.

Cohen EE, Rosen LS, Vokes EE, Kies MS, Forastiere AA, Worden FP, Kane MA, Sherman E, Kim S, Bycott P et al. 2008 Axitinib is an active treatment for all histologic subtypes of advanced thyroid cancer: results from a phase II study. Journal of Clinical Oncology 26 4708-4713. (doi:10.1200/JCO.2007.15.9566)

De Souza JA, Busaidy N, Zimrin A, Seiwert TY, Villaflor VM, Poluru KB, Reddy PL, Nam J, Vokes EE \& Cohen EE 2010 Phase II trial of sunitinib in medullary thyroid cancer (MTC). Proceedings of the American Society of Clinical Oncology 285504.

Eisenhauer EA, Therasse P, Bogaerts J, Schwartz LH, Sargent D, Ford R, Dancey J, Arbuck S, Gwyther S, Mooney M et al. 2009 New response evaluation criteria in solid tumours: revised RECIST guideline (version 1.1). European Journal of Cancer 45 228-247. (doi:10.1016/j. ejca.2008.10.026)

Gupta-Abramson V, Troxel AB, Nellore A, Puttaswamy K, Redlinger M, Ransone K, Mandel SJ, Flaherty KT, Loevner LA, O'Dwyer PJ et al. 2008 Phase II trial of sorafenib in advanced thyroid cancer. Journal of Clinical Oncology 26 4714-4719. (doi:10.1200/JCO.2008.16.3279)

Hoftijzer H, Heemstra KA, Morreau H, Stokkel MP, Corssmit EP, Gelderblom H, Weijers K, Pereira AM, Huijberts M, Kapiteijn E et al. 2009 Beneficial effects of sorafenib on tumor progression, but not on radioiodine uptake, in patients with differentiated thyroid carcinoma. European Journal of Endocrinology 161 923-931. (doi:10.1530/EJE-09-0702)

Kloos RT, Ringel MD, Knopp MV, Hall NC, King M, Stevens R, Liang J, Wakely PE Jr, Vasko VV, Saji M et al. 2009 Phase II trial of sorafenib in metastatic thyroid cancer. Journal of Clinical Oncology 27 1675-1684. (doi:10.1200/JCO.2008.18.2717)

Kurzrock R, Cohen EE, Sherman SI, Pfister DG, Cohen RB, Ball D, Hong DS, Ng CS, Salgia R \& Ratain MJ 2010 Long-term results in a cohort of medullary thyroid cancer
(MTC) patients (pts) in a phase I study of XL184 (BMS 907351), an oral inhibitor of MET, VEGFR2, and RET. ASCO Meeting Abstracts 285502.

Kurzrock R, Sherman SI, Ball DW, Forastiere AA, Cohen RB, Mehra R, Pfister DG, Cohen EE, Janisch L, Nauling F et al. 2011 Activity of XL184 (Cabozantinib), an oral tyrosine kinase inhibitor, in patients with medullary thyroid cancer. Journal of Clinical Oncology 29 2660-2666. (doi:10. 1200/JCO.2010.32.4145)

Lam ET, Ringel MD, Kloos RT, Prior TW, Knopp MV, Liang J, Sammet S, Hall NC, Wakely PE Jr, Vasko VV et al. 2010 Phase II clinical trial of sorafenib in metastatic medullary thyroid cancer. Journal of Clinical Oncology 28 2323-2330. (doi:10.1200/JCO.2009.25. 0068)

Leboulleux S, Bastholt L, Krause TM, De La Fouchardiere C, Tennvall J, Awada A, Gómez JM, Tisseron-Carrasco A, Licour M \& Schlumberger MJ 2010 Vandetanib in locally advanced or metastatic differentiated thyroid cancer (papillary or follicular; DTC): a randomized, double-blind phase II trial. Annals of Oncology (ESMO Meeting Abstracts) 21 viii314-viii328.

Nikiforova MN \& Nikiforov YE 2008 Molecular genetics of thyroid cancer: implications for diagnosis, treatment and prognosis. Expert Review of Molecular Diagnostics 8 83-95. (doi:10.1586/14737159.8.1.83)

Puxeddu E, Romagnoli S \& Dottorini ME 2011 Targeted therapies for advanced thyroid cancer. Current Opinion in Oncology 23 13-21. (doi:10.1097/CCO. Ob013e328340cf94)

Ravaud A, de la Fouchardiere C, Courbon F, Asselineau J, Klein M, Nicoli-Sire P, Bournaud C, Delord J, Weryha G \& Catargi B 2008 Sunitinib in patients with refractory advanced thyroid cancer: the THYSU phase II trial. ASCO Meeting Abstracts 266058.

Schlumberger MJ, Elisei R, Bastholt L, Wirth LJ, Martins RG, Locati LD, Jarzab B, Pacini F, Daumerie C, Droz JP et al. 2009 Phase II study of safety and efficacy of motesanib in patients with progressive or symptomatic, advanced or metastatic medullary thyroid cancer. Journal of Clinical Oncology 27 3794-3801. (doi:10. 1200/JCO.2008.18.7815)

Sherman SI, Wirth LJ, Droz JP, Hofmann M, Bastholt L, Martins RG, Licitra L, Eschenberg MJ, Sun YN, Juan T et al. 2008 Motesanib diphosphate in progressive differentiated thyroid cancer. New England Journal of Medicine 359 31-42. (doi:10.1056/NEJMoa075853)

Sherman SI, Jarzab B, Cabanillas ME, Licitra LF, Pacini F, Martins R, Robinson B, Ball D, McCaffrey J, Shah MH et al. 2011 A phase II trial of the multi-targeted kinase inhibitor, lenvatinib (E7080), in advanced radioiodine (RAI)-refractory differentiated thyroid cancer (DTC). ASCO Meeting Abstracts 295503.

Therasse P, Arbuck SG, Eisenhauer EA, Wanders J, Kaplan RS, Rubinstein L, Verweij J, Van Glabbeke M, van Oosterom AT, Christian MC et al. 2000 New guidelines to 
evaluate the response to treatment in solid tumors. Journal of the National Cancer Institute 92 205-216. (doi:10.1093/jnci/92.3.205)

Wells SA Jr, Gosnell JE, Gagel RF, Moley J, Pfister D, Sosa JA, Skinner M, Krebs A, Vasselli J \& Schlumberger M 2010 Vandetanib for the treatment of patients with locally advanced or metastatic hereditary medullary thyroid cancer. Journal of Clinical Oncology 28 767-772. (doi:10.1200/JCO.2009.23.6604)

Wells SA Jr, Robinson BG, Gagel RF, Dralle H, Fagin JA, Santoro M, Baudin E, Elisei R, Jarzab B, Vasselli JR et al. 2011 Vandetanib in patients with locally advanced or metastatic medullary thyroid cancer: a randomized, double-blind phase III trial. Journal of Clinical Oncology 30 134-141. (doi:10.1200/JCO.2011.35.5040)

Wilhelm SM, Carter C, Tang L, Wilkie D, McNabola A, Rong H, Chen C, Zhang X, Vincent P, McHugh M et al. 2004 BAY 43-9006 exhibits broad spectrum oral antitumor activity and targets the RAF/MEK/ERK pathway and receptor tyrosine kinases involved in tumor progression and angiogenesis. Cancer Research 64 7099-7109. (doi:10.1158/0008-5472.CAN04-1443)

Yu XM, Lo CY, Chan WF, Lam KY, Leung P \& Luk JM 2005 Increased expression of vascular endothelial growth factor $\mathrm{C}$ in papillary thyroid carcinoma correlates with cervical lymph node metastases. Clinical Cancer Research 11 8063-8069. (doi:10.1158/ 1078-0432.CCR-05-0646)

Received in final form 19 January 2012 Accepted 27 January 2012 Made available online as an Accepted Preprint 27 January 2012 